\title{
Measurements and simulations of wakefields at the Accelerator Test Facility 2
}

\author{
J. Snuverink, ${ }^{1, *}$ R. Ainsworth, ${ }^{1}$ S. T. Boogert, ${ }^{1}$ F. J. Cullinan, ${ }^{1}$ A. Lyapin, ${ }^{1}$ \\ Y. I. Kim, ${ }^{2,3}$ K. Kubo, ${ }^{4,5}$ S. Kuroda, ${ }^{4,5}$ T. Okugi, ${ }^{4,5}$ T. Tauchi, ${ }^{4,5}$ N. Terunuma, ${ }^{4,5}$ \\ J. Urakawa, ${ }^{4,5}$ and G. R. White ${ }^{6}$ \\ ${ }^{1}$ John Adams Institute at Royal Holloway, University of London, Surrey TW2O OEX, United Kingdom \\ ${ }^{2}$ John Adams Institute at University of Oxford, Oxford OX1 3RH, United Kingdom \\ ${ }^{3}$ Center for Axion and Precision Physics Research, Institute for Basic Science (IBS), 291, Daehak-ro, \\ Yuseong-gu, Daejeon, Republic of Korea \\ ${ }^{4}$ KEK, Ibaraki 305-0801, Japan \\ ${ }^{5}$ School of High Energy Accelerator Science, SOKENDAI, Ibaraki 305-0801, Japan \\ ${ }^{6}$ SLAC, Menlo Park, California 94025, USA
}

(Received 19 August 2015; revised manuscript received 18 June 2016; published 15 September 2016)

\begin{abstract}
Wakefields are an important factor in accelerator design, and are a real concern when preserving the low beam emittance in modern machines. Charge dependent beam size growth has been observed at the Accelerator Test Facility (ATF2), a test accelerator for future linear collider beam delivery systems. Part of the explanation of this beam size growth is wakefields. In this paper we present numerical calculations of the wakefields produced by several types of geometrical discontinuities in the beam line as well as tracking simulations to estimate the induced effects. We also discuss precision beam kick measurements performed with the ATF2 cavity beam position monitor system for a test wakefield source in a movable section of the vacuum chamber. Using an improved model independent method we measured a wakefield kick for this movable section of about $0.49 \mathrm{~V} / \mathrm{pC} / \mathrm{mm}$, which, compared to the calculated value from electromagnetic simulations of $0.41 \mathrm{~V} / \mathrm{pC} / \mathrm{mm}$, is within the systematic error.
\end{abstract}

DOI: 10.1103/PhysRevAccelBeams.19.091002

\section{INTRODUCTION}

Wakefields are increasingly important in the context of precision beam operation and control. The problem of wakefield measurement and simulation has been a standing item in the design of circular machines for decades. It is now equally important for single pass accelerators due to stringent requirements imposed by the luminosity (colliders) or brightness (free electron lasers) specifications. Sometimes it is necessary or desirable to measure wakefields of some components in situ in an existing beam line. This can be done by fitting the beam orbit to a known beam line model for each pass of the beam in order to reduce the influence of the beam jitter [1,2].

Here we demonstrate an improved method of measuring wakefields in single pass beam lines using model independent methods for jitter subtraction that do not require prior knowledge of the reference orbit or the beam optics. Using this method a large pulse-to-pulse orbit jitter can be effectively subtracted. In combination with a submicrometer resolution cavity beam position monitor

\footnotetext{
*Jochem.Snuverink@ rhul.ac.uk

Published by the American Physical Society under the terms of the Creative Commons Attribution 3.0 License. Further distribution of this work must maintain attribution to the author(s) and the published article's title, journal citation, and DOI.
}

(CBPM) system, the orbit reconstruction in a beam test carried out at the Accelerator Test Facility 2 (ATF2) also reached submicrometer precision, allowing us to characterize relatively weak wakefield sources.

\section{A. Accelerator Test Facility 2}

ATF2 [3] is a scaled demonstrator for the local chromaticity correction scheme [4] proposed for the future linear lepton colliders for focusing the beams to nanometer sizes at the interaction point (IP) and reaching the desired luminosity [5,6]. This scheme's most notable advantage is the considerable reduction in length of the final focus system compared to other proposals.

ATF2 is built as an extension of the ATF damping ring at KEK (Japan). The beam from a low emittance damping ring is extracted into the ATF2 beam line (Fig. 1). The main parameters of the extracted beam are summarized in Table I.

The evolution of the Twiss parameters throughout the length of the ATF2 beam line is shown in Fig. 2. After some preparation and diagnostics, the beam is focused by the final focus system, producing a small size at the IP, defined as the focal point of the last focusing quadrupole, where the beam size is measured. The beam size is measured directly using a beam size ("Shintake") monitor, which uses crossed laser beams to produce sub-beam-size interference patterns 


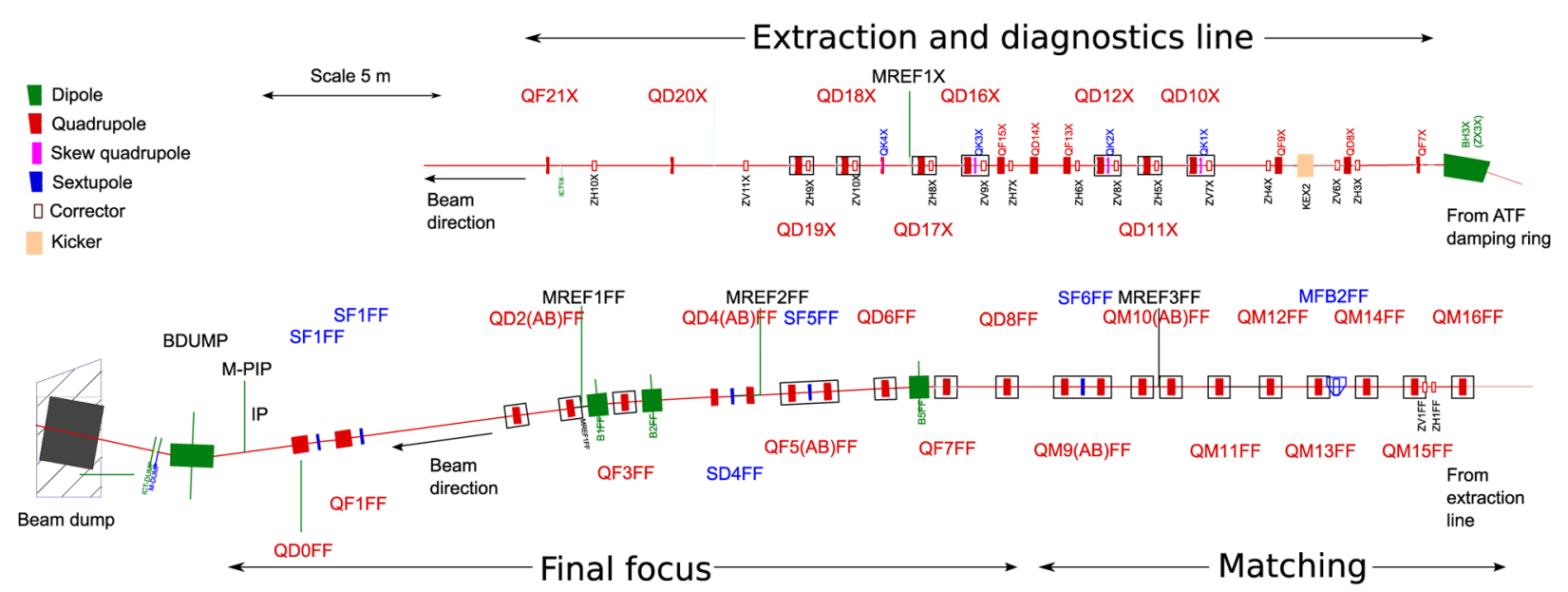

FIG. 1. Schematic diagram of the ATF2 beam line, taken from [7].

at the IP. The electron beam inverse Compton scatters with the laser photons producing a detectable gamma ray signal and the interference pattern can be translated by phase shifting one of the laser beams [8].

The aim of the ATF2 is twofold: first, to obtain and verify a vertical focus size of $37 \mathrm{~nm}$; second, to hold the focus stable to within a few nanometers [3,9]. Maintaining the small vertical beam emittance throughout the beam line is therefore essential. The ATF2 collaboration has routinely measured a beam size below $100 \mathrm{~nm}$ vertically, although only at a bunch charge of $0.1 \times 10^{10}$ electrons per bunch [10]. Since efforts are concentrated towards demonstrating small beam sizes in the vertical plane, most of the discussions in this paper focus on the vertical axis.

Achieving a vertical beam size below $100 \mathrm{~nm}$ required lowering the bunch charge from the nominal by a factor of 10. This had a positive side effect of reducing the Compton signal background of several beam diagnostics. However, achieving the second goal will require all the beam diagnostics to operate at the highest resolution only possible at higher beam charges.

One of the main contributions to beam size growth at higher charges is thought to be from transverse wakefields. The ATF2 beam line includes a number of high impedance elements, such as cavity beam position monitors, bellows, vacuum ports and step transitions. Normally, the impact of wakefield sources on the beam is negligible for single-pass

TABLE I. Nominal beam parameters for ATF2.

\begin{tabular}{lccc}
\hline \hline Parameter & Symbol & Value & Units \\
\hline Beam energy & $\mathrm{E}$ & 1.30 & $\mathrm{GeV}$ \\
Horizontal emittance & $\epsilon_{x}$ & 2 & $\mathrm{~nm} \mathrm{rad}$ \\
Vertical emittance & $\epsilon_{y}$ & 12 & $\mathrm{pm} \mathrm{rad}$ \\
Bunch length & $\sigma_{z}$ & $7-9$ & $\mathrm{~mm}$ \\
Electrons per bunch & $N_{e}$ & $1 \times 10^{10}$ & $e^{-}$ \\
Fractional momentum spread & $\Delta p / p$ & 0.001 & \\
Typical beam pipe radius & $\mathrm{a}$ & 12.0 & $\mathrm{~mm}$ \\
\hline \hline
\end{tabular}

beam lines. However, the ATF2 beam is especially sensitive to wakefields (an order of magnitude more than expected at the ILC) due to the long bunch lengths at ATF2, 7-9 mm, and relatively low beam energy.

Normally, transverse wakefield effects can be mitigated by introducing orbit bumps. However, this approach is not suitable in the ATF2 final focus beam line, where the beam orbit needs to be adjusted precisely for making an extremely small beam at the IP. Any beam position change in the sextupole magnets (at high beta-function locations) will induce significant higher order aberrations.

The technique adopted at ATF2 includes mitigating the wakefield sources and compensating the wakes that cannot be mitigated using a movable section of the beam line. Wake compensation is now part of the ATF2 beam tuning procedure and has been crucial in achieving beam sizes below $100 \mathrm{~nm}$.

This paper covers the wakefield studies that contributed to the results reported in [10] (prior to spring 2013).
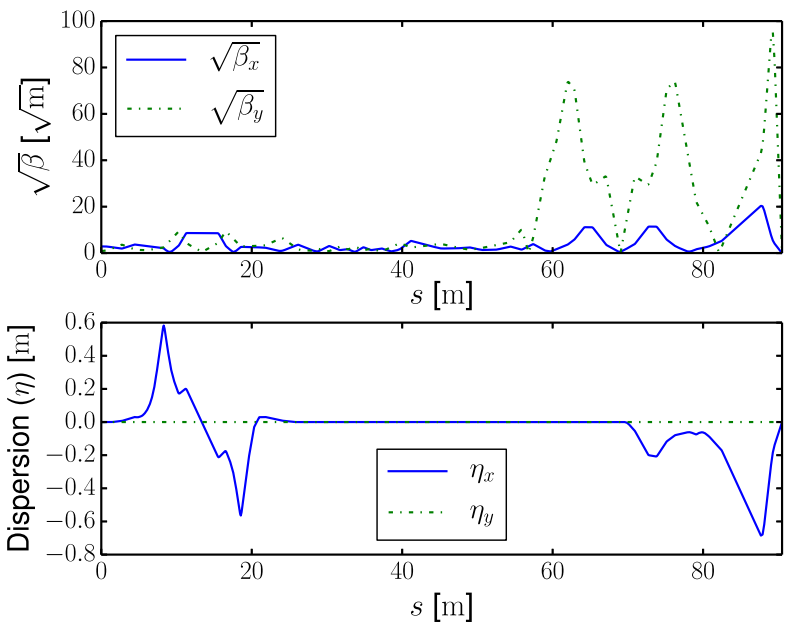

FIG. 2. Twiss parameters for the nominal ATF2 lattice as a function of the longitudinal coordinate $s$. 
We identify some of the major wakefield sources and establish a method of simulating and measuring the wakefield kicks.

\section{B. Wakefields}

We consider wakefields that are produced when the electromagnetic (EM) fields surrounding a bunch of charged particles interact with geometrical discontinuities in the beam line, called geometric wakefields. Besides wasting the energy stored in the beam, the produced EM waves may affect the following bunches, and the bunch itself, provided it is long enough to "see" these fields as they travel at the speed of light (the "catch-up" effect). The effects of the wakefields on the longitudinal and transverse beam motion are usually expressed as integral characteristics, called wake potentials [11]. Here we are primarily interested in the transverse wake potential: The longitudinal wake potential introduces $\mathrm{keV}$ level distortions of the energy that do not affect the $1.3 \mathrm{GeV}$ ATF2 beam strongly enough to produce any visible effect at the IP. By contrast, transverse wakefields have been shown to produce noticeable effects, most importantly on the measured beam size. Transverse wakefields alter the orbit of a bunch as a whole (i.e. center of mass kick) according to its total charge and transverse position with respect to the wakefield generating elements; and also introduce an orbit modulation along the bunch length (tilt), so the particles along the length of the bunch arrive with slightly different offsets at the IP, perceived as a beam size increase.

In general, the transverse wakefield $W$ depends on the three-dimensional charge distribution in the bunch. Here we consider a pencil beam since the transverse beam size is small compared to the aperture throughout the beam line. As a smaller beam size target is set for the vertical direction, all wake effect simulations and observations were done for the vertical direction.

Then, the wake potential $W(z, \bar{y})$ is a function of the longitudinal position in the bunch $z$ and the vertical offset of the beam position with respect to the wakefield source $\bar{y}$. For small offsets, assuming the structure has a symmetry axis, it can be assumed to be linear with the offset: $W(z, \bar{y}) \approx \bar{y} W_{n}(z)$.

If $W_{n}(z)$ is the transverse wake potential created by the bunch normalized by the transverse offset $\bar{y}$ in a source at a location $a$ in the beam line, and $\rho(z)$ its longitudinal charge distribution, then the vertical center of mass position offset $\Delta y$ at a location $b$ downstream will depend on the optics between these locations as well as the beam parameters [12]:

$$
\Delta y \approx R_{34, a \rightarrow b} \frac{e \bar{y}}{E} \int_{-\infty}^{\infty} W_{n}(z) \rho(z) \mathrm{d} z,
$$

where $E$ is the beam energy, and $R_{34, a \rightarrow b}$ the 3-4 component of the transfer matrix between $a$ and $b . R_{34, a \rightarrow b}$ can be calculated from the beta functions $\beta_{a, b}$ and betatron phases $\phi_{a, b}$ using the following equation:

$$
R_{34, a \rightarrow b}=\sqrt{\beta_{a} \beta_{b}} \sin \left(\phi_{b}-\phi_{a}\right) .
$$

Hence, the wakefield-induced offsets can be significant at locations with large beta functions.

To quantify the average kick a bunch of particles receives, we define the weighted average wake potential $W_{\text {avg }}(y)$ :

$$
\begin{aligned}
W_{\mathrm{avg}}(\bar{y}) & =\frac{\int_{-\infty}^{\infty} W(z, \bar{y}) \rho(z) \mathrm{d} z}{\int_{-\infty}^{\infty} \rho(z) \mathrm{d} z} \\
& \approx \frac{\bar{y} \int_{-\infty}^{\infty} W_{n}(z) \rho(z) \mathrm{d} z}{\int_{-\infty}^{\infty} \rho(z) \mathrm{d} z}=\kappa_{\perp} \bar{y},
\end{aligned}
$$

where $\kappa_{\perp}$ is known as the dipole kick factor. We also define the normalized peak wake potential:

$$
W_{n, \text { peak }}=\max _{z}\left|W_{n}(z)\right| .
$$

\section{WAKEFIELD SIMULATIONS}

\section{A. Wakefield calculations}

A number of ATF2 beam line elements have been investigated. The wake potential produced by their modeled geometries was simulated primarily using the EM simulation code GdfidL [13]. GdfidL runs a finite difference loop to numerically propagate the beam excited EM fields on a cubic mesh. The beam is represented by a line charge with a Gaussian distribution along the $z$-axis, and offsets from the beam axis can be specified in both vertical and horizontal directions.

Wakefield kick measurements described in the following sections started with the C-band reference cavities, hence we chose to consider them here in some detail. The geometry, as meshed by GdfidL, is shown in Fig. 3. It features a cylindrical cavity connected to a cylindrical beam pipe. Although the C-band reference cavity has an asymmetric arrangement for coupling the signals out, the difference of vertical and horizontal wakefields for the first few oscillations is small (in the order of a few percent), and has been neglected.

As a computational cross-check, we also calculated the wake potentials for this geometry using a second code, $\mathrm{T} 3 \mathrm{P}$ from the ACE3P suite of codes [14], which uses curvilinear tetrahedral meshing. The transverse wake potential for various beam offsets is shown in Fig. 4, where GdfidL results are plotted in solid lines and T3P in dashed lines. It can be seen that there is a very good agreement between the results produced by the two codes. It is also interesting that the wake potential has a pronounced nonlinear behavior with respect to the beam offset in the part that coincides with the bunch excitation $(z<0.016 \mathrm{~m}$ in Fig. 4$)$, while 


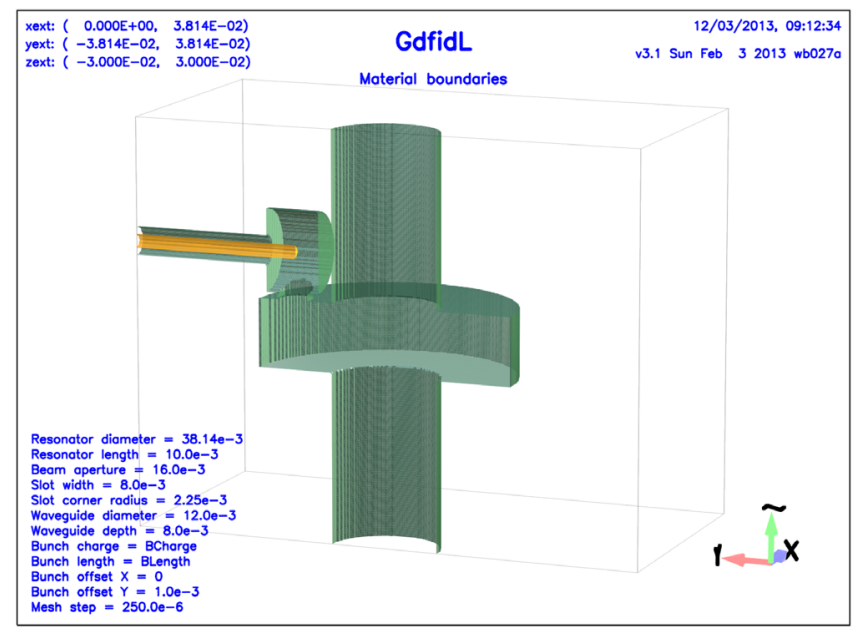

FIG. 3. GdfidL C-band reference cavity model (sliced at the symmetry plane).

retaining the linearity after the bunch transit. This is most likely caused by differences in field configurations allowed in the cavities with and without the beam present, i.e. electrostatic fields accompanying the beam versus excited cavity modes.

A third order polynomial has been fitted to the weighted average values of the wake potential at different offsets (Fig. 5). The third order coefficient of $-1.6 \times$ $10^{-3} \mathrm{~V} / \mathrm{pC} / \mathrm{mm}^{3}$ has little effect near the cavity center compared to the linear term, corresponding to the dipole mode wake of $-0.08 \mathrm{~V} / \mathrm{pC} / \mathrm{mm}$ (compared to $-0.09 \mathrm{~V} / \mathrm{pC} / \mathrm{mm}$ calculated from a single offset of $1 \mathrm{~mm}$ ), but becomes significant for large beam offsets.

Figure 4 also shows the charge distribution used for calculating the excitation. One can see that the wake

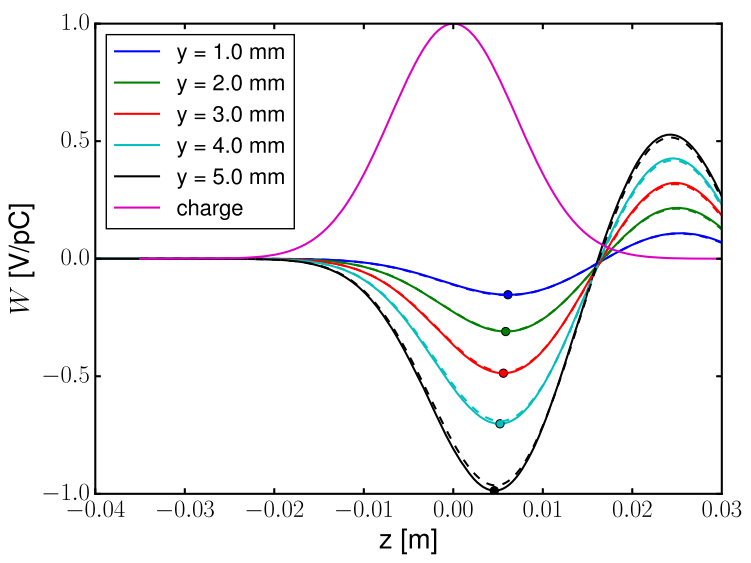

FIG. 4. The wake potential produced by a $7 \mathrm{~mm}$ long bunch traveling with different offsets in the vertical direction in the $\mathrm{C}$-band reference cavity. The centered Gaussian shape shows the charge distribution used in calculations, where $z<0$ corresponds to the head of the bunch. The solid lines are calculated with GdfidL and the dashed lines with T3P. The dots indicate the position of the peak wake potential.

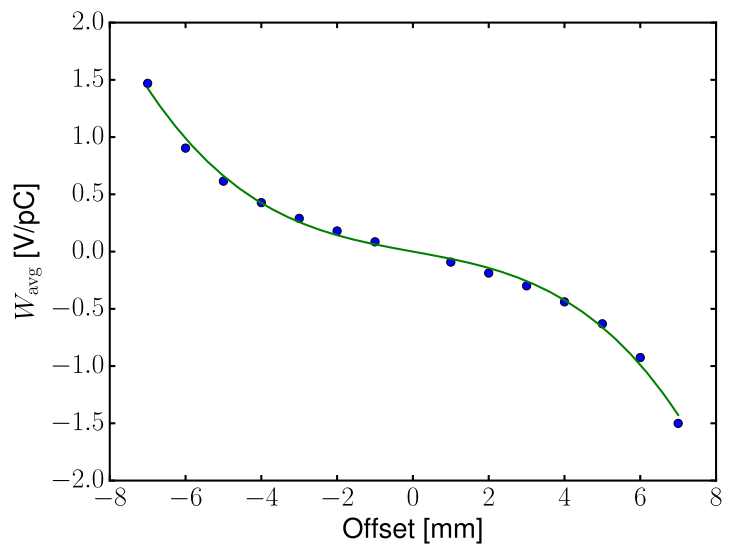

FIG. 5. Weighted average wake potential of the C-band reference cavity as a function of beam offset fitted to a third order polynomial.

potential peaks while the bunch still passes the cavity, and that the wake potential changes substantially on the time scale of the bunch passage. Hence, both overall kick and bunch tilt are to be expected. Other beam line components (see Table II) showed similar wake potential shapes with the peak more or less delayed depending on the discontinuity dimensions.

Table II summarizes the values of the peak normalized wake potential $W_{n \text {,peak }}$ and the weighted average normalized wake potential $W_{n \text {,avg }}$ for the beam line components that have been studied so far in the order they are most likely to affect the beam, taking into account the quantity of installed objects as of spring 2013. The wake potentials were evaluated at a fixed beam offset of $1 \mathrm{~mm}$, except for the bellows, where the beam remained centered while the offset was introduced between the two ends of the beam pipe.

The simulations indicate that shields must be used for the vacuum bellows as their wakefields may reach the level of wakes produced in CBPMs at the same offset, but their alignment is typically much poorer, a similar argument applies to the vacuum flanges, especially the ones with aperture steps, due to their high numbers.

TABLE II. Summary of the peak and weighted average normalized wake potential calculated from a single offset of $1 \mathrm{~mm}$ for a bunch length of $7 \mathrm{~mm}$. The quantities indicated are the approximate situation in spring 2013.

\begin{tabular}{lccc}
\hline \hline Component & $\begin{array}{l}\left|W_{n, \text { peak }}\right| \\
\mathrm{V} / \mathrm{pC} / \mathrm{mm}\end{array}$ & $\begin{array}{c}\left|W_{n, \text { avg }}\right| \\
\mathrm{V} / \mathrm{pC} / \mathrm{mm}\end{array}$ & Quantity \\
\hline Bellows & 0.1 & 0.06 & $\sim 100$ \\
Vacuum flanges + step & 0.06 & 0.04 & $\sim 100$ \\
Vacuum flanges & 0.03 & 0.02 & $\sim 100$ \\
C-band position & 0.11 & 0.06 & $\sim 40$ \\
C-band reference & 0.15 & 0.09 & 4 \\
Vacuum ports & 0.07 & 0.05 & 6 \\
\hline \hline
\end{tabular}




\section{B. Tracking simulations}

Especially for this study, an option for including simulated wakefields imported from field solvers has been integrated and published into the particle tracking code PLACET [15]. This code incorporates single bunch and multibunch effects, static and dynamic imperfections. In PLACET a distinction is made between wakefields acting on the same bunch and wakefields acting on the following bunch, called short-range and long-range wakefields respectively. For this study a new way of describing a short-range wakefield (transverse and/or longitudinal) has been added for each beam line component. The new description consists of a spline that describes the normalized wake potential $W_{n}(z)$, with units $\mathrm{V} / \mathrm{mm} / \mathrm{pC}$ as a function of the longitudinal coordinate $z$ in meters [15]. This implementation can be directly used with GdfidL output, for example as given in Fig. 4. The beam is modeled by a set of Gaussian-distributed macroparticles within the beam ellipse. When the beam interacts with the wakefield the kick is calculated by interpolating the spline for each macroparticle at position $z$, analog to Eq. (1) the kick for the vertical direction $\Delta y^{\prime}(z)$ is given by

$$
\Delta y^{\prime}(z)=W_{n}(z) \frac{e \bar{y} N}{E},
$$

with $N$ the number of particles in the bunch.

As will be described in Sec. III B, a movable section of vacuum chamber was installed in the ATF2 beam line near the quadrupole magnet QD10BFF located in a high betatron function location to compensate the wakefield distortion to the beam and measure the orbit response. This setup has been added to the ATF2 lattice simulations and with the help of the modified PLACET software the vertical differential orbit response has been analyzed. The response expected at various beam intensities for a vertical offset of the setup of $1 \mathrm{~mm}$ is shown in Fig. 6. The average position of the beam

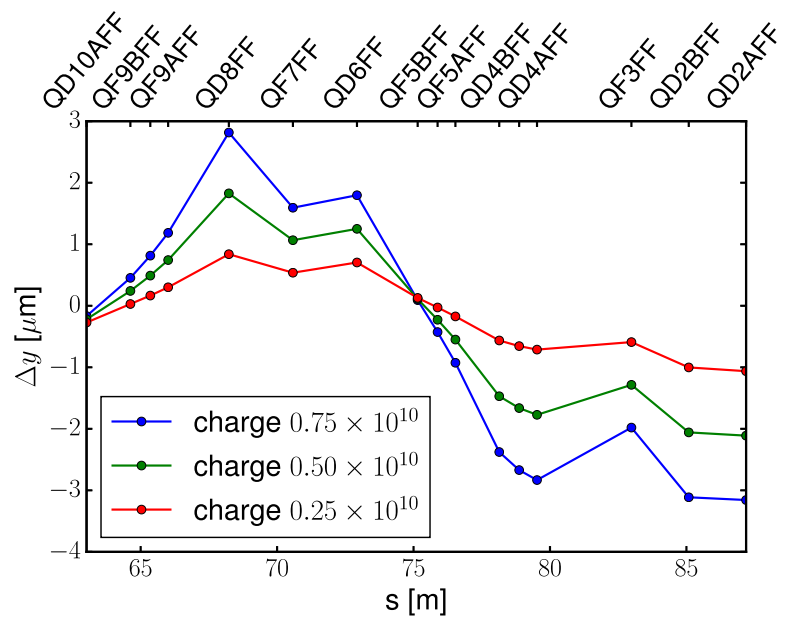

FIG. 6. Simulated vertical orbit response for a $1 \mathrm{~mm}$ move of the movable section before quadrupole magnet QD10BFF (described in Sec. III B). is plotted for each CBPM location downstream of the movable section. As expected, the orbit change corresponds to the beta function amplitude at the CBPM location.

\section{ORBIT MEASUREMENTS}

\section{A. Cavity beam position monitor system}

The position measurement system at the ATF2 provides the precision required for measuring wakefield-induced beam offsets of the order of a micrometer. The system currently includes a total of 39 CBPMs. The first ten CBPMs are mounted in fixed quadrupoles, the next 25 are mounted in quadrupoles which are moved by three axis movers and the four IP region BPMs (two normal C-band and two especially designed for the IP) are rigidly fixed, but not in magnets. The beam line also includes several reference cavities providing an independent charge and phase reference for the position measurement. The majority of CBPMs are operated at a resolution of $200 \mathrm{~nm}$ in a $\pm 1 \mathrm{~mm}$ range with $20 \mathrm{~dB}$ front-end attenuation, with several providing $30 \mathrm{~nm}$ without attenuation [7].

\section{B. Experimental setup}

To study the beam distortion and orbit change induced by the wakefields and also to investigate the possibility of compensation of the wakefields generated by other sources, a movable test section has been installed in the ATF2 beam line. The movable section includes a two axis mover system with a range of $\pm 4.5 \mathrm{~mm}$ in both vertical and horizontal directions. The system is located $62.5 \mathrm{~m}$ downstream of the extraction kicker in a high betatron amplitude location, $\beta_{y}=5000 \mathrm{~m}$, between the quadrupoles QD10BFF and QD10AFF. The setup analyzed in this paper includes two C-band reference cavities in series as in Fig. 7 also

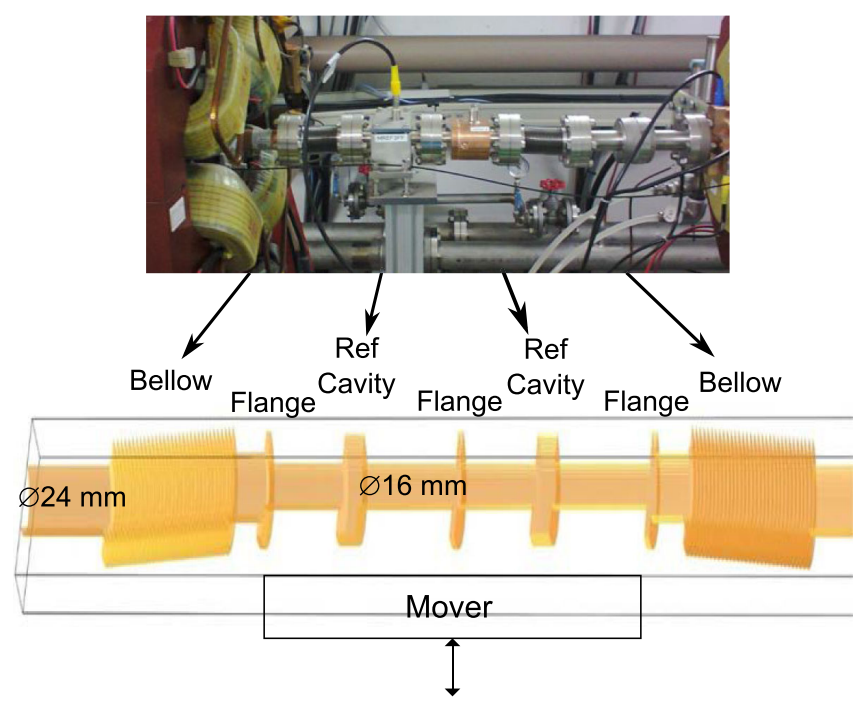

FIG. 7. Wakefield experimental installation with two C-band reference cavities, vacuum flanges and two bellows. The full 3D GdfidL model of the installation is also shown. 


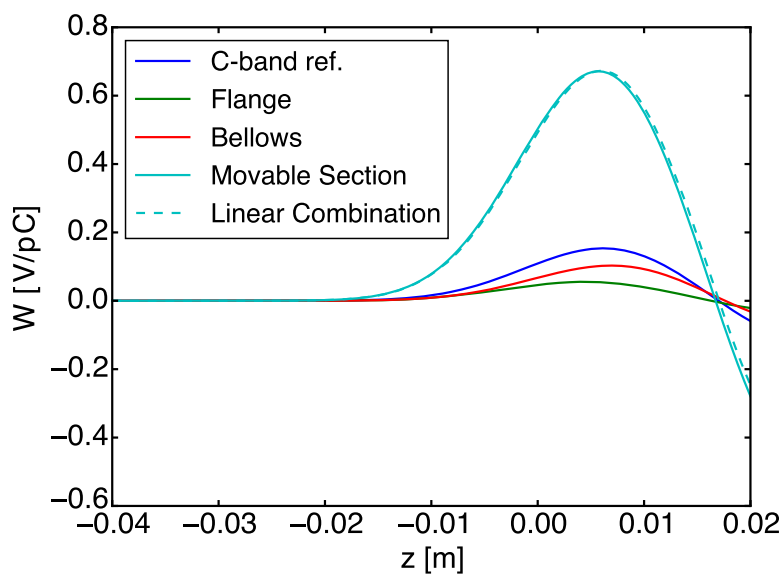

FIG. 8. The transverse wake potential of the movable section and its components for a $1 \mathrm{~mm}$ translation and its individual components produced by a $7 \mathrm{~mm}$ long bunch, where $z<0$ corresponds to the head of the bunch.

showing two bellows and aperture steps (from $\emptyset 24$ to $\emptyset 16 \mathrm{~mm}$ ) at both sides of the cavities as well as flanges after the bellows and in between the cavities. The whole section has been 3D modeled in GdfidL and the model is also shown in Fig. 7.

The bellows on both sides of the cavities are deformed as the mover position changes. In the simulations it had been assumed that the bellows deform linearly during the movement.

The wakefield contributions of the individual components and the whole section are shown in Fig. 8. The linear combination of the individual components in the section (two reference cavities, three flanges and two bellows) is also shown and agrees well with the whole section simulation. The peak potential for the whole movable section is $0.67 \mathrm{~V} / \mathrm{mm} / \mathrm{pC}$ and the total weighted average wakefield contribution comes to about $0.41 \mathrm{~V} / \mathrm{mm} / \mathrm{pC}$.

\section{Jitter subtracted orbit measurement}

The wakefield induced orbit change is measured by the CBPMs downstream of the movable section, while CBPMs upstream provide data for projecting the orbit and subtracting the effect of the incoming beam jitter. Since the betatron function is higher in the vertical direction in the downstream beam line, and hence the wakefields would produce larger offsets vertically, typically a vertical mover position scan was performed. During the data taking, about 100-200 machine pulses for each position of the wakefield source were recorded.

The pulse-to-pulse orbit jitter, which is up to tens of $\mu \mathrm{m}$ vertically (depending on the betatron value) is subtracted by correlating the readings of the upstream and downstream CBPMs. The correlation matrix $\mathbf{X}$ of $n_{1}$ (27) upstream CBPMs with $n_{2}$ (16) downstream CBPMs is defined as
$\mathbf{A X}=\mathbf{B}$

with matrix A (B) containing the upstream (downstream) average subtracted CBPM readings for all $m$ pulses recorded at the nominal mover position. Matrix $\mathbf{X}$ is then determined in a least-square sense by inverting matrix $\mathbf{A}$ with the singular value decomposition method, with a relative cut on the small singular values of $1 \times 10^{-4}$. The residual matrix $\mathbf{R}$ (of size $n_{2} \times m$ ) is calculated as

$$
\mathbf{R}=\mathbf{A} \mathbf{X}-\mathbf{B} .
$$

The residuals calculated for the data with no mover section offset indicate the precision of the orbit reconstruction for each of the downstream CBPMs. In Fig. 9 the standard deviation of the residuals is shown for each downstream BPM for data with an average bunch charge of $0.75 \times 10^{10}$ particles. It can be seen that the orbit reconstruction is well below a micrometer for each BPM. The same operation was applied to the data at different mover positions using the same matrix $\mathbf{X}$. Here, the residuals reveal the difference between the predicted and measured orbit at each location with the jitter subtracted.

For each pulse, the absolute charge reading was recorded based on the measurement of the inductive current transformer (ICT) in the extraction beam line. With this charge information, the residuals for each individual pulse are normalized to the average charge of all pulses in the mover position scan. Then the residuals are averaged for each downstream CBPM location and mover position to extract the systematic offsets.

In Fig. 10 the residuals are plotted with respect to the position of the movable section at a downstream location where the orbit response is one of the strongest, in this case the CBPM near quadrupole QD2BFF. The average bunch charge was measured as $0.75 \times 10^{10}$ particles. The

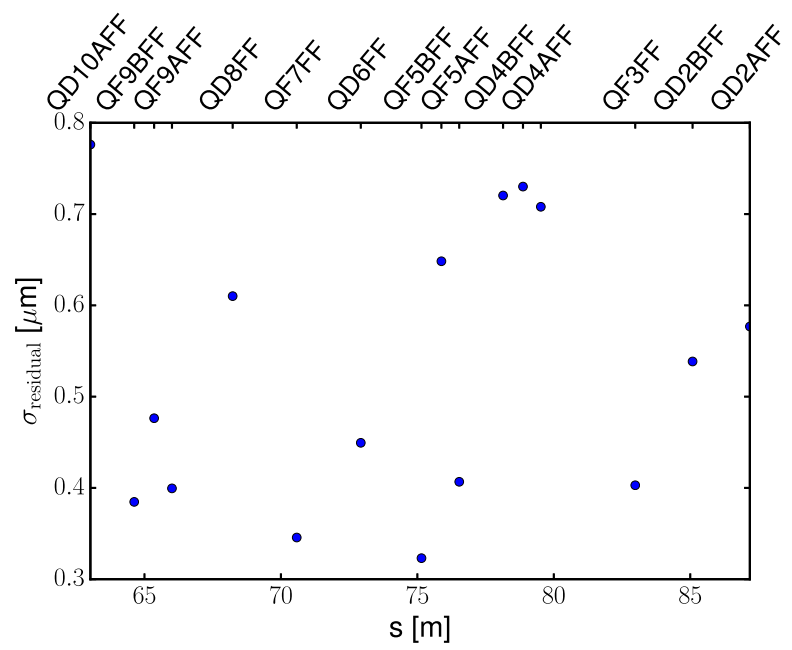

FIG. 9. Standard deviation of the residuals for all downstream BPMs. 


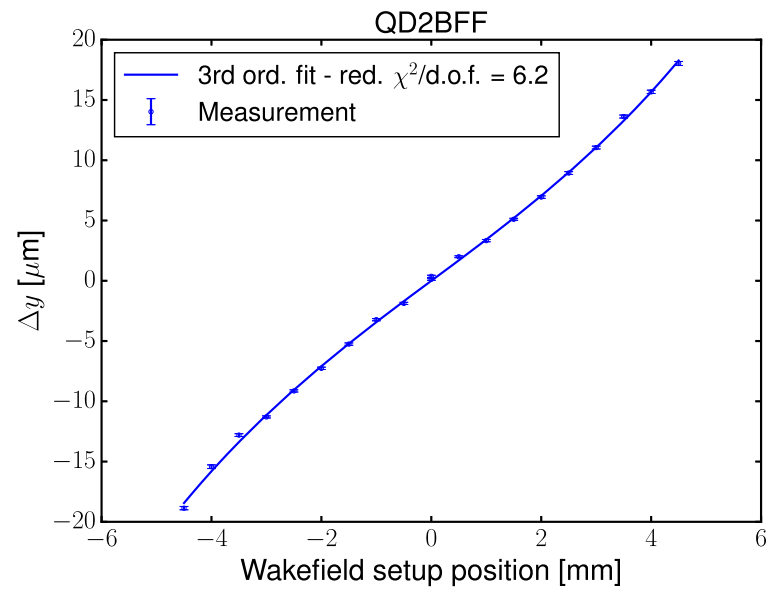

FIG. 10. The orbit change for an average bunch charge of $0.75 \times 10^{10}$ particles with respect to the wakefield section position after pulse averaging and jitter subtraction of the CBPM readings in the vertical direction near quadrupole QD2BFF.

dependence is very clear with a statistical error on each point of about $200 \mathrm{~nm}$. The data is fitted to a third order polynomial as predicted by the numerical calculations with a $\chi^{2} /$ d.o.f. of 6.2 . The third order component is clearly present. At the location of QD2BFF shown in Fig. 10 the vertical beam orbit is changed by $3.2 \mu \mathrm{m}$ per each $\mathrm{mm}$ of the source translation (in the linear region). We define a sensitivity parameter $k$ as the constant of proportionality between the source position and average beam movement at a downstream BPM.

Another signature feature of the presence of wakefields is the intensity dependence of the kicks. To confirm this, the measurements were repeated for different bunch intensities. The intensity is obtained by averaging the charge reading from the ICT. In Fig. 11 the vertical orbit response for different bunch intensities is shown for each downstream

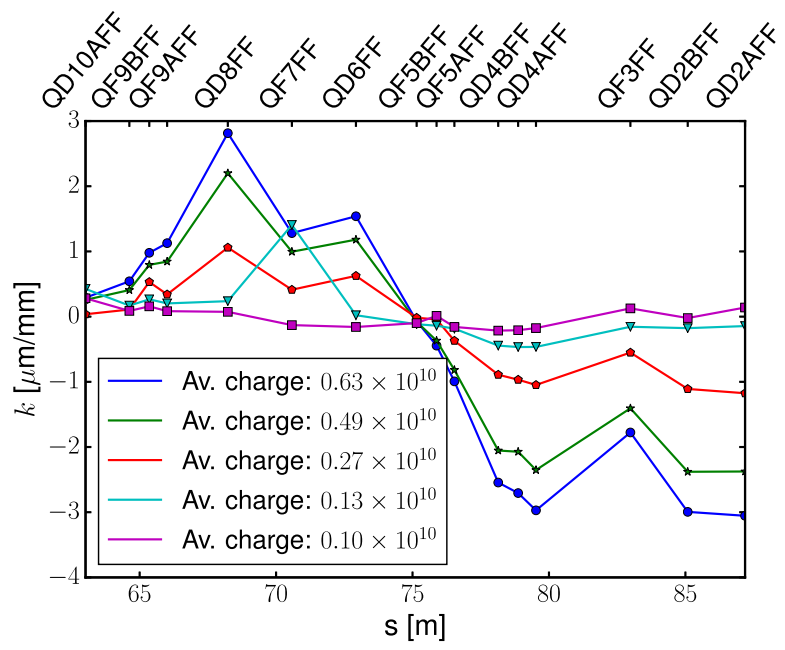

FIG. 11. The vertical orbit response for each CBPM with respect to the movable section position for different bunch intensities after pulse averaging and jitter subtraction.

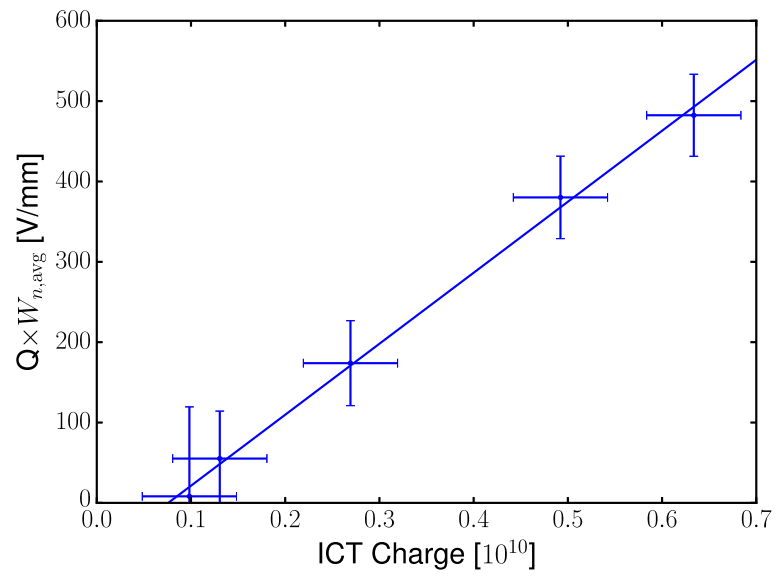

FIG. 12. The normalized weighted average wake potential of the vertical orbit response with respect to the movable section position for different bunch intensities after pulse averaging and jitter subtraction. A linear fit is shown as well.

CBPM and in Fig. 12 the normalized weighted average wake potential of the movable section calculated with the tracking simulation of Fig. 6 and the orbit response of all downstream CBPMs of Fig. 11 is shown for each bunch intensity. A clear linear intensity dependence has been observed. Note that the CBPM resolution is degraded at lower bunch charges [7] and hence the precision of the jitter subtraction and the orbit response measurement is also lower.

\section{Combined wakefield and optics studies}

For each of the downstream CBPMs the response with respect to the mover position has been determined, for an average bunch charge of $0.75 \times 10^{10}$ particles, and compared with the results of tracking simulations described in Sec. II B, this is shown in Fig. 13.

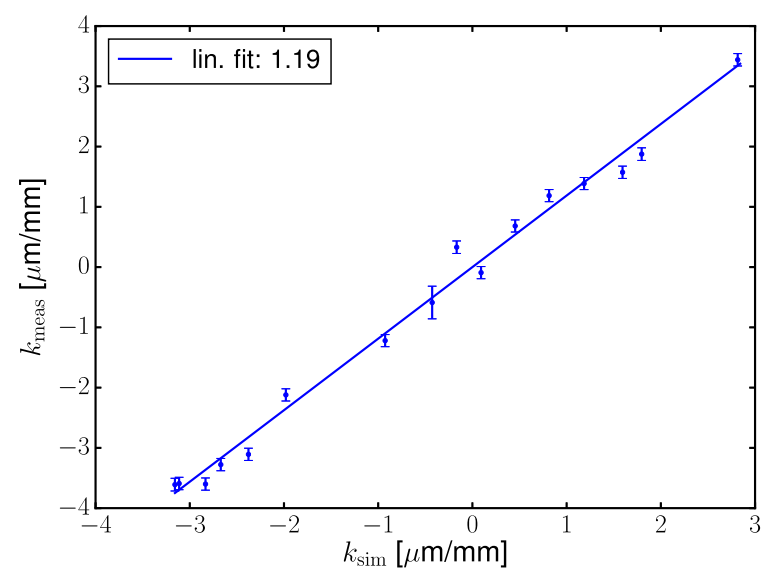

FIG. 13. The vertical orbit response for each CBPM with respect to the movable section position for the movable section compared to the simulation for an average bunch charge of $0.75 \times 10^{10}$ particles. The measurement and simulation are compared after the pulse averaging and jitter subtraction. A linear fit is shown as well. 
The simulation shows a good agreement with the observed orbit change. The measured response appears to be $20 \%$ higher than the prediction, so that the measurement corresponds to an average wakefield potential of about $0.49 \mathrm{~V} / \mathrm{pC} / \mathrm{mm}$ compared to the estimate of $0.41 \mathrm{~V} / \mathrm{pC} / \mathrm{mm}$ based on numerical calculations.

\section{E. Discussion}

The improved model independent method allowed us to subtract the beam jitter efficiently from the response due to the wakefield excitation along the beam line. There are further advantages in the proposed method: (i) The precision of the orbit reconstruction reaches that of the position diagnostics meaning that weaker sources can be characterized or a shorter beam line is required for detecting the same kick. (ii) The matrices used for the model independent jitter subtraction can be extended to include, for example, seismic sensors to reduce uncorrelated jitter, or temperature or other environmental data in case drifts are observed.

The measured wakefield of the movable section is about $20 \%$ higher than the prediction based on numerical calculations. Taking into account that the calculations for the deformed bellows and vacuum flanges were based on approximate geometries, this result is reasonable. Additionally, some beam conditions were not entirely certain: the bunch length and shape are measured in the damping ring to a reasonable precision of about half a $\mathrm{mm}$, which corresponds to a wakefield strength uncertainty of $5 \%-10 \%$. They, however, are not monitored in the ATF2 extraction beam line, where the section is actually located. It is also possible that the charge measurement contributes about $5 \%-10 \%$ uncertainty due to calibration error of the ICT. The precision of the beam optics model may also contribute, although the ATF2 beam line is well understood, so this contribution should be small in comparison, and further reduced due to the large number of measurement locations.

Following our study electromagnetic shielding was added to most of the bellows, numerous unnecessary connections, aperture steps and components were removed or modified. The component alignment has also been revisited several times in order to reduce the average beam offset throughout the ATF2 beam line.

The type of problem explained in this paper is of a limited significance for future linear colliders [12] due to shorter compressed bunch lengths. In the case of the ILC the wakefield kicks were estimated to be 10 times lower compared to ATF2 [12]. Still, the methodology outlined in this paper can be applied in existing and future beam lines, where wakefield induced kicks may be present, in particular free electron lasers. Furthermore, long range intratrain wakefield effects will still be a problem in future linear colliders and the method presented is also applicable for a bunch train.

\section{CONCLUSIONS}

Several wakefield sources have been characterized by putting them in a movable section, of which the two C-band reference cavity combination has been presented here. The measurements have been performed with the cavity BPM system, which is operating with a typical resolution of $200 \mathrm{~nm}$. The peak wakefield estimated from the combination of the measurement and tracking simulation is about $0.49 \mathrm{~V} / \mathrm{pC} / \mathrm{mm}$, which is $20 \%$ larger than the calculated value from electromagnetic simulations of $0.41 \mathrm{~V} / \mathrm{pC} / \mathrm{mm}$, where the discrepancy is most likely down to some uncertainties in the conditions of the experiment, such as the exact composition and behavior during mechanical translation of the section itself, and the shape and length of the extracted bunch.

\section{ACKNOWLEDGMENTS}

The research leading to these results has received funding from the Science and Technology Facilities Council via the John Adams Institute, Royal Holloway University of London. We would like to acknowledge CERN for financial support of this research within CLICUK collaboration: Contract No. KE1870/DG/CLIC. This work was supported in part by Department of Energy Contract No. DE-AC02-76SF00515. This work was supported by IBS-R017-D1-2016-a00.

[1] P. Tenenbaum et al., Direct measurement of the transverse wakefields of tapered collimators, Report No. SLAC-PUB12086, 2007.

[2] J. L. Fernandez-Hernando et al., Measurements of collimator wakefields at End Station A, in Proceedings of the 11th European Particle Accelerator Conference, Genoa, 2008 (EPS-AG, Genoa, Italy, 2008), WEPP163.

[3] ATF2 Proposal, KEK Report No. 2005-2.

[4] P. Raimondi and A. Seryi, Novel Final Focus Design for Future Linear Collider, Phys. Rev. Lett. 86, 3779 (2001).

[5] T. Behnke et al., arXiv:1306.6327. See also http://www .linearcollider.org/ILC/TDR for a full list of contributing institutes.

[6] M. Aicheler et al., A multi-TeV linear collider based on CLIC technology, 2012, doi:10.5170/CERN-2012-007.

[7] Y. I. Kim et al., Cavity beam position monitor system for the Accelerator Test Facility 2, Phys. Rev. ST Accel. Beams 15, 4 (2012).

[8] J. Yan et al., Measurement of nanometer electron beam sizes with laser interference using Shintake monitor, Nucl. Instrum. Methods Phys. Res., Sect. A 740, 131 (2014).

[9] A. Seryi et al., ATF2 commissioning, in Proceedings of the 23rd Particle Accelerator Conference, Vancouver, Canada, 2009 (IEEE, Piscataway, NJ, 2009), p. 4205.

[10] G. R. White et al., Experimental Validation of a Novel Compact Focusing Scheme for Future Energy-Frontier 
Linear Lepton Colliders, Phys. Rev. Lett. 112, 034802 (2014).

[11] P. B. Wilson, Introduction to wakefields and wake potentials, Report No. SLAC-PUB-4547, 1989.

[12] K. Kubo, A. Lyapin, and J. Snuverink, Wakefield issues for the linear colliders, Beam Dynamics Newsletter 61, 69 (2013).

[13] W. Bruns, GdfidL: A finite difference program with reduced memory and CPU usage, in Proceedings of the Particle
Accelerator Conference, Vancouver, BC, Canada, 1997 (IEEE, New York, 1997), p. 2651, http://www.gdfidl.de.

[14] K. Ko et al., Advances in parallel electromagnetic codes for accelerator science and development, Report No. SLAC-PUB-14349, 2011.

[15] A. Latina et al., Evolution of the tracking code PLACET, in IPAC2013: Proceedings of the 4th International Particle Accelerator Conference (JACoW, CERN, Geneva, Switzerland, 2013), p. 1014. 